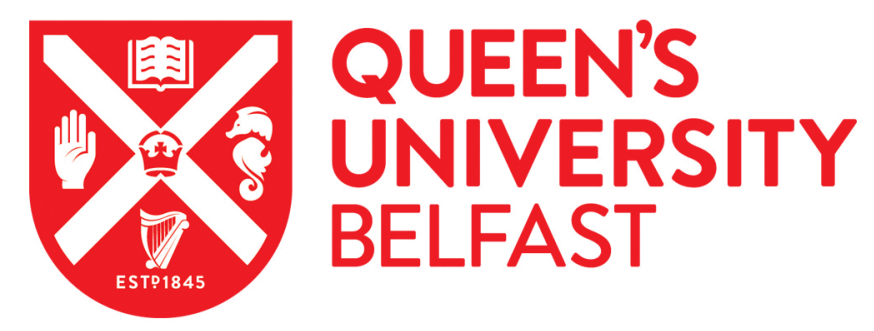

\title{
Meditation and Endocrine Health and Wellbeing
}

Pascoe, M. C., Thompson, D. R., \& Ski, C. F. (2020). Meditation and Endocrine Health and Wellbeing. Trends in Endocrinology \& Metabolism, 31(7), 469-477. https://doi.org/10.1016/j.tem.2020.01.012

\section{Published in:}

Trends in Endocrinology \& Metabolism

\section{Document Version:}

Peer reviewed version

\section{Queen's University Belfast - Research Portal:}

Link to publication record in Queen's University Belfast Research Portal

\section{Publisher rights}

Copyright 2020 Elsevier.

This manuscript is distributed under a Creative Commons Attribution-NonCommercial-NoDerivs License

(https://creativecommons.org/licenses/by-nc-nd/4.0/), which permits distribution and reproduction for non-commercial purposes, provided the author and source are cited.

\section{General rights}

Copyright for the publications made accessible via the Queen's University Belfast Research Portal is retained by the author(s) and / or other copyright owners and it is a condition of accessing these publications that users recognise and abide by the legal requirements associated with these rights.

Take down policy

The Research Portal is Queen's institutional repository that provides access to Queen's research output. Every effort has been made to ensure that content in the Research Portal does not infringe any person's rights, or applicable UK laws. If you discover content in the Research Portal that you believe breaches copyright or violates any law, please contact openaccess@qub.ac.uk. 


\section{Opinion}

Meditation and Endocrine Health and Wellbeing

Michaela C. Pascoe ${ }^{1 *}$, David R. Thompson ${ }^{2,3}$ and Chantal F. Ski ${ }^{2,3}$

${ }^{1}$ Institute of Sport, Exercise and Active Living, Victoria University, Melbourne, Australia

${ }^{2}$ Department of Psychiatry, University of Melbourne, Melbourne, Australia

${ }^{3}$ School of Nursing and Midwifery, Queen's University Belfast, Belfast, United Kingdom

* Correspondence: Michaela.pascoe@vu.edu.au

Key words: Meditation, Endocrine, Health, Wellbeing

Grant support: There is no funding to report 


\section{Abstract}

Meditation is a popular practice for reducing stress and improving mental health and wellbeing. Its effects are mediated largely by the endocrine system, including the hypothalamic-pituitary-adrenal axis, the hypothalamic-pituitary-thyroid axis and the renin-angiotensin-aldosterone system, and energy homeostasis. The limited evidence available indicates that changes associated with endocrine function following meditation correspond with improvements in mental health. However, this field of study is hampered by a lack of consensus as to definition and types of meditation and the mixed quality of reported studies. Moreover, the exact mechanisms by which meditation operates remain unclear and more robust studies are required to explore this by delineating the target populations, forms, dosages and modes of delivery of meditation, comparison groups, and health experiences and outcomes used.

\section{Highlights}

There is increasing interest in the practice of meditation and its effects on physiological markers of stress, meditated largely by the endocrine system, though the precise links and mechanisms by which these occur remain unclear.

Most studies have investigated the effects of meditation practice on the hypothalamic-pituitaryadrenal axis, with comparatively little attention paid to other parts of the endocrine system.

Growing but limited evidence indicates that changes associated with endocrine function after meditation may correspond with improvements in mental health outcomes, but more robust definitions of meditation and studies to demonstrate and explain its effects are required. 


\section{Glossary}

Adrenocorticotropin hormone: A polypeptide tropic hormone that is an important component of the hypothalamic-pituitary-adrenal axis and is often produced in response to biological stress

Corticotropin-releasing hormone: A peptide hormone involved in the stress response

Cortisol: A glucocorticoid hormone widely used biomarker of HPA axis dysfunction

Endocrine system: A number of glands that produce hormones which regulate many functions in the body and is important in managing and responding to stress

Hypothalamic-Pituitary-Adrenal (HPA) Axis: A neuroendocrine system that controls reactions to stress and regulates many body processes

Hypothalamic-Pituitary-Thyroid (HPT) Axis: An endocrine system that regulates thyroid hormone production

Insulin: A peptide hormone necessary for the control of blood glucose

Leptin: A hormone secreted by and in proportion to adipose cells and regulates energy by inhibiting hunger

Meditation: Practices and techniques, such as mindfulness, transcendental meditation, and breath awareness that encourage and develop concentration, clarity, emotional positivity and a cultivation of non-judgmental awareness

Renin-Angiotensin-Aldosterone (RAA) System: An endocrine system that regulates blood pressure, electrolyte and fluid balance

Stress: A disruption to homeostasis and stressor-induced activation of the sympathetic nervous system and 'stress response', which protects the body in the short term and regulates adaptation 


\section{Meditation Influences the Stress Response}

Meditation, which has its origins in India and dates as far back as $5000 \mathrm{BCE}$, has become increasingly popular and widely practiced as a secular and therapeutic activity [1]. The word meditation stems from meditatum, a Latin term 'to ponder'. Meditation practices are techniques, such as mindfulness, transcendental meditation, and breath awareness that encourage and develop concentration, clarity, emotional positivity and a cultivation of non-judgmental awareness [2, 3]. Various forms spiritual and secular - of meditation have been developed, with the latter emphasizing stress reduction and relaxation [4]. However, the term meditation has been used to designate a variety of practices that differ enough from each other that they elude precise definition [4], and the lack of consensus in defining meditation has hampered its acceptance for study in the scientific community. Nevertheless, research on meditation has increased dramatically over the last 50 years. Studies in the West have explored the impact of meditation on mental and physiological outcomes, and though the exact mechanisms at work remain elusive there is evidence that following meditation changes are seen in brain structure and function e.g. thickening of the cerebral cortex, lower frequency alpha and theta waves [5-7], physiological markers of stress e.g. lower blood cortisol levels, slower respiratory rate $[8,9]$ and cardiovascular risk factors e.g. lower blood pressure and heart rate [10], mediated largely by the endocrine system and in response to stressors.

\section{The Endocrine System and Stress}

The endocrine system comprises a number of glands - the hypothalamus, pituitary gland and pineal gland in the brain, the thyroid and parathyroid glands in the neck, the thymus in between the lungs, the adrenal glands on top of the kidneys, the pancreas behind the stomach and the gonads in the pelvic region - that produce hormones which regulate many functions in the body and is important in managing and responding to stress. It is a chemical messenger system comprising of feedback loops regulated by hormones released by glands into the circulatory system to regulate distant organs, often mediated via the hypothalamus and pituitary [11]. 
Stress can be defined as disruption to homeostasis and activation of the stress response, or 'fight-orflight' response. Humans, as with all living things, maintain a dynamic equilibrium - homeostasis which is the stable state or balance of the organism and of optimal functioning. Humans are also constantly challenged by internal or external stressors, which can be real or perceived threats to homeostasis (or safety or wellbeing) and result in adaptive physiological and metabolic changes that maximise chances of surviving these threats [12]. This stress response is mainly regulated by the central nervous system through activation of the sympathetic and parasympathetic divisions of the autonomic nervous system and actions of neuro-humoral mediators such as the hypothalamicpituitary-adrenal axis and the catecholaminergic system [13]. This includes increases in heart rate and blood pressure to send oxygen to muscles, pupil dilation to let in as much light as possible, and the downstream release of the glucocorticoid hormone, cortisol, from the adrenal cortex, as well as suppression of nonessential systems, like the digestive and immune systems, to allow more energy for emergency functions [12]. Once the threat has receded the parasympathetic nervous system returns the body to homeostasis [13].

Stress induced physiological and metabolic changes are crucial for survival in the presence of an actual present-moment threat but can have a range of negative effects when activated unnecessarily and chronically. This is because stressors can be both real and perceived, and psychological stressors such as worry about the future, anticipation, reliving the past, rumination and arousal can lead to chronic activation of the stress response, which is detrimental to health and wellbeing as it increases the risk of health problems and contributes to a wide variety of diseases, disorders and difficulties [14]. Indeed, worry and anticipation about the future and reliving the past are associated with activation of the default mode network (DMN), a large-scale network of interacting brain regions, most commonly shown to be active when the brain is at wakeful rest, when an individual is thinking about others, themselves, remembering the past, and planning for the 
future $[15,16]$. Alterations in the DMN activity have been associated with a number of mental health problems, including depression and anxiety [16], which are characterised by high DMN activity such as worry and rumination $[17,18]$. Mindfulness practices, specifically various forms of meditation, which bring the person back to the present moment have been associated with reduced activity in the DMN $[19,20]$.

Given the above, it is not surprising that persistent activation of the stress response is associated with the onset and maintenance of mental health issues such as anxiety and depression [14, 21, 22], perhaps resulting, at least in part, from disruption in the feedback mechanisms required to return the body to homeostasis once a threat has passed. Stress-related increases in glucocorticoid levels and the further synthesis of pro-inflammatory cytokines (small cell signalling protein molecules involved in the innate immune response and inflammation) [23] are normally regulated by a glucocorticoid negative feedback mechanism [24]. However, persistent activation of the stress response disrupts this negative feedback mechanism [24] and results in a cumulative physiological burden which can eventually contribute to the onset of disease, mental illness and poor wellbeing $[14,25,26]$. As the neuroendocrine systems regulating the stress response are involved in the regulation of mood and emotion [27], mental illnesses such as clinical anxiety and depression are associated with increased expression of stress-induced pro-inflammatory cytokines [23], that stimulate the autonomic nervous system and hyper-secretion of corticotropin-releasing hormone $(\mathrm{CRH})$, and increase circulatory cortisol and the production of pro-inflammatory cytokines $[23,24$, $28,29]$.

While the endocrine system is important in the management of stress, the effects of meditation on the functioning of the endocrine system and wellbeing have been scarcely investigated [30-32]. In regards to the impact of meditation on the human endocrine system, the hypothalamic-pituitaryadrenal axis has been most widely explored. 


\section{Meditation Influences the Hypothalamic-Pituitary-Adrenal (HPA) Axis}

The HPA axis is a neuroendocrine system that is involved in stress regulation and various other bodily processes and plays a key role in mental health and wellbeing. The HPA axis controls the synthesis and release of stress hormones including $\mathrm{CRH}$, adrenocorticotropin hormone (ACTH), and cortisol, in response to stressors. The release of $\mathrm{CRH}$ from the hypothalamus results in release of ACTH, which then acts on the adrenal cortex to release cortisol into the blood (Figure 1). Cortisol subsequently acts in a negative feedback fashion to terminate the continued release of $\mathrm{CRH}$. Disruptions in cortisol production can result in dysregulation of the HPA axis, which has been associated with a range of mental health problems, such as depression and anxiety [32].

The HPA axis has been the mostly widely studied of the endocrine systems in regards to the effects of meditation. A systematic review of 45 randomized controlled trials investigating the effects of focused attention, open monitoring and automatic self-transcending subtypes of meditation (defined in Box 1) on markers of stress, and compared to an active control group, found that focused attention, but not automatic self-transcending meditation, reduced cortisol levels [9]. In this review [9], meta-analysis to investigate the effects of open monitoring meditations, such as mindfulness meditation, was unable to be performed due to an insufficient number of trials. Previous research, however, indicates that mindfulness-based meditation may also influence HPA axis stress hormone levels, and that this is associated with improved mental health and wellbeing [33-35]. For example, in a pre-post study of 16 individuals with clinical depression and anxiety, two months of mindfulness meditation improved psychological wellbeing and increased ACTH, indicating that mindfulness meditation can enhance psychological well-being and regulate hormonal parameters [31]. In a randomized controlled trial of 150 healthy individuals, practising amrita meditation four times a week resulted in a decline in adrenaline and cortisol levels from as early as 48 hours after beginning the meditation program. The decline in adrenaline and cortisol levels was similarly present after eight months of engaging in amrita meditation four times a week, compared to progressive muscle 
relaxation and a no intervention control [36]. In cross-sectional studies of regular transcendental meditation (a form of automatic self-transcending mediation) practitioners (three to five years of practice) and new transcendental meditation practitioners (three to four months of practice), the former group were associated with more marked and sustained declines in cortisol levels than the latter group [37, 38]. In a study of 34 Chinese undergraduate students, two and four weeks of integrative body-mind training, which included mindfulness training, reduced salivary cortisol levels both at rest and following a laboratory-based stress-inducing task, compared to relaxation [39].

In a pre-post study of 59 patients with early stage breast or prostate cancer, a mindfulness-based stress reduction program, which included meditation, was associated with decreased cortisol levels as well as levels of pro-inflammatory cytokines, systolic blood and stress symptoms, indicating less stress and mood disturbance [40]. In a pilot study of 30 postmenopausal women, salivary and urinary excretion of cortisol following a metabolic stressor (oral glucose consumption) was higher in 16 long-term practitioners of transcendental meditation when compared with 14 non-meditators, which may reflect improved endocrine regulation in response to metabolic challenge [41]. In a randomized controlled trial of 57 patients with colorectal cancer, a single mindfulness meditation practice delivered during active chemotherapy administration resulted in increased cortisol reactivity, suggesting that mindfulness practice can reduce the blunting of neuroendocrine profiles typically observed in cancer patients and supporting the use of mindfulness in oncology [42]. These studies collectively indicate that meditation influences the regulation of the HPA axis, which may reflect decreased stress levels among meditators. 
Box 1 Delineation of Meditation Types

\begin{tabular}{|l|l|}
\hline $\begin{array}{l}\text { Open } \\
\text { monitoring }\end{array}$ & $\begin{array}{l}\text { Open monitoring or mindfulness-based meditation involves non-reactive } \\
\text { observation of the content of ongoing experience, to become reflectively aware } \\
\text { of cognitive and emotional patterns }[43,44] .\end{array}$ \\
\hline $\begin{array}{l}\text { Focused } \\
\text { attention }\end{array}$ & $\begin{array}{l}\text { In focused attention meditation, attention is focused and sustained on a particular } \\
\text { object and brought back to the object when the mind has wandered. Thus, the } \\
\text { meditator is controlling one's own attention [43-45]. }\end{array}$ \\
\hline $\begin{array}{l}\text { Automatic } \\
\text { self- } \\
\text { transcending }\end{array}$ & $\begin{array}{l}\text { AST involves a meaningless mantra that the meditator can attend to without } \\
\text { effort or concentration, with the aim of the mantra becoming secondary and } \\
\text { ultimately disappearing as self-awareness increases. In AST meditation the mind } \\
\text { should be free from focus and mental effort [46]. }\end{array}$ \\
\hline
\end{tabular}

\section{Meditation Influences the Hypothalamic-Pituitary-Thyroid (HPT) Axis}

The HPT axis determines and regulates thyroid hormone production, which is vital to metabolism, nervous system development and thermogenesis, among other functions [47], and appears to be involved in the pathophysiology of anxiety [48] and depression [49]. Thyroid-stimulating hormone (TSH) controls the synthesis and release of triiodothyronine (T3) and thyroxine (T4). Plasma levels of $\mathrm{T} 3$ and T4 are maintained within a narrow range and too much or too little can lead to hyperthyroidism or hypothyroidism, respectively (Figure 1) [47]. There is limited scientific research regarding the effects of meditation on thyroid hormones, though the available evidence suggests that some forms of meditation may influence thyroid functioning in particular populations, as discussed below.

With age, TSH levels gradually increase, possibly due to decreased biological activity of the peptide hormone, or increasing thyroid resistance to TSH. However, in long-term practitioners of transcendental meditation, TSH levels are seen to decrease [50]. This is apparent in a prospective study of 11 men who had been practising transcendental meditation for at least three years, where an advanced transcendental meditation practice (termed TM-Sidhi) was associated with a decline in TSH levels, but not in cortisol, T4 or T3 levels, indicating that transcendental meditation may effect 
some, but not all, markers of neuroendocrine function [50]. In a randomized controlled trial of 49 healthy Caucasian males, four months of transcendental meditation practice, 15-20 minutes twice a day, was associated with a decrease in TSH and cortisol levels and an increase in growth hormone levels, compared with stress education transcendental meditation [51]. It is possible that transcendental meditation was associated with a decrease in cortisol levels in this study as individuals were meditation naive at baseline [51], whereas in the earlier study [50], as individuals had already been practising transcendental meditation for at least three years, any changes in cortisol levels had already occurred [51]. A more recent non-randomized study of 45 healthy men and women found that a 12 week yoga program including an unspecified form of meditation increased TSH levels in men, and decreased T3 and T4 levels in both men and women, compared to a waitlist control group [52]. It is possible that a yoga program including physical postures may differ from meditation alone in terms of effects on thyroid function, as physical yogic postures can influence HPT axis function $[53,54]$. This is consistent with research showing that other forms of physical activity and exercise influence HPT axis function. For example, in a study involving six rowers, three weeks of high-intensity resistance training decreased TSH and T3 levels, while three weeks of endurance training increased TSH levels [55]. In obese women, TSH levels have been shown to increase during and decrease immediately after 60 minutes of aerobic exercise, while TSH levels increase and T3 levels decrease after three months of aerobic exercise training [56]. These studies collectively suggest that meditation as well as yoga including meditation may influence TSH levels, albeit in differing ways, and perhaps that these effects may be sex-specific. This hypothesis is supported by findings that in 20 middle-aged sedentary women, an eight week yoga program that included an unspecified form of meditation did not influence TSH, T4 or T3 levels, compared to a nointervention control group [57], indicating that the yoga/meditation intervention did not influence thyroid function. In sixteen individuals (nine female) with elevated depression and anxiety symptoms, two months of mindfulness meditation practised at least three times a week similarly did not influence TSH, T3 and T4 levels, though this study did not assess males and females separately 
[31]. In a pilot study of 22 women with hypothyroidism, however, six months of yoga practice four times a week, including cyclic meditation, resulted in a non-significant reduction in TSH, cholesterol, triglycerides, low density lipoprotein and high-density lipoprotein levels [58]. These results indicate that meditation may improve thyroid function in women with thyroid dysfunction more effectively than in women with normal thyroid function, or that interventions of longer duration are required in order to detect benefits following meditation interventions. In addition, it should also be noted that only those studies delivering transcendental meditation were found to decrease TSH levels, and therefore different forms of meditation may also account for the divergence in results. Overall, the limited evidence suggests that some forms of meditation may influence thyroid function, which may reflect more efficient functioning of the HPT axis, and that these effects may be sex- or populationspecific.

\section{[INSERT Figure 1 here]}

\section{Meditation Influences the Renin-Angiotensin-Aldosterone (RAA) System}

The RAA system regulates blood pressure, electrolyte and fluid balance [59]. Renin is an aspartic protease protein and enzyme that converts angiotensinogen to angiotensin I. Angiotensin I is converted to angiotensin II, a potent vasoconstrictive peptide, by angiotensin converting enzyme (ACE) and acts on the adrenal resulting in the release of aldosterone, a steroid hormone that increases blood pressure by causing the kidney to retain water [59]. Angiotensin II is also an important stress hormone and increases following acute and chronic stress [60]. Given its role in stress, it is not surprising that aldosterone and renin are also associated with psychological wellbeing. In a cross sectional study of 1743 individuals, living alone in combination with depressive symptomatology was seen to be associated with increased renin and aldosterone levels, while neither living alone nor having depressive symptomatology alone were associated with changes in 
renin and aldosterone levels, indicating that depressed individuals may have an activated RAA system during potentially stressful circumstances [61].

While limited, the existing evidence indicates that meditation may influence the RAA system as well as stress outcomes. For example, in a cross sectional study of eight male college students who had been practising transcendental meditation for at least two years, plasma renin activity increased during transcendental meditation, compared to a quite rest condition [62]. Interestingly these meditators had a smaller increase in cortisol following venepuncture, compared to people who were not meditators, indicating that transcendental meditation may reduce stress reactivity [62]. Similarly, a cross-sectional study of 22 healthy students who had practised transcendental meditation for 8.5 years found that, compared to 33 non-meditators, they had lower levels of aldosterone, cortisol, and excretion of the norepinephrine metabolite vanillylmandelic acid, and higher levels of the serotonin metabolite, 5-hydroxyindoleacetic acid, which corresponded with lower levels of mood disturbance and anxiety [38]. This indicates that meditation-associated changes in the RAA system correspond with improved well-being and changes in hormonal stress markers [38].

\section{Meditation Influences Energy Homeostasis}

Energy homeostasis depends on the balance between energy intake and expenditure. The physiological control of energy homeostasis involves multiple mechanisms and physiological systems and organs, such as the brain and white adipose tissues [63]. The brain integrates satiety or hunger signals and regulates the insulin response of glucose metabolism, among other functions [64]. There are few existing studies exploring the influence of meditation on energy homeostasis. This review briefly highlights the findings of identified studies on insulin resistance and leptin. 


\section{Meditation and Insulin Resistance}

Insulin is a peptide hormone considered to be the main anabolic hormone and is necessary for the control of blood glucose levels as it signals the liver, muscle and fat cells to uptake glucose from the blood to be used for energy [65]. A recent statement from the American Heart Association highlights that there is limited research regarding the effects of meditation on metabolism and insulin resistance [10].

In a pre-post study of 50 individuals with type II diabetes, a single session of a sitting breathing meditation was associated with reduced levels of postprandial plasma glucose and systolic and diastolic blood pressure [66], though these measures were taken directly after completing the intervention and may reflect acute rather than longer term changes.

In regards to longer term interventions, in a randomized controlled trial of 103 individuals with coronary artery disease, 16 weeks of transcendental meditation was associated with improved insulin resistance (and systolic blood pressure and heart rate variability), compared to health education [67]. However, in a pre-post study of 37 individuals with (30\%) and without (70\%) coronary artery disease, six weeks of yoga and mindfulness meditation for 90 minutes three times a week was not associated with a significant reduction in blood glucose, lipid and C-reactive protein levels in either group [68], indicating that transcendental meditation may influence metabolism more effectively than mindfulness meditation.

\section{Meditation and Leptin}

Leptin is a hormone secreted by and in proportion to adipose cells and regulates energy by inhibiting hunger. Leptin plays a role in inflammatory process; specifically as increases in leptin are associated with an increase in C-reactive protein [69]. The limited data indicate that meditation does not 
appear to influence leptin levels. For example, in a randomized controlled trial of 186 university faculty and staff with an elevated C-reactive protein level, and with or at risk of cardiovascular disease, a two-month workplace mindfulness program incorporating mindfulness meditation, compared to lifestyle education, was not associated with any changes in leptin [70]. Similar results were found in a randomized controlled trial of 68 African American individuals who had high metabolic system risk factors, as 12 months of consciously resting meditation, which is a sound or mantra based meditation, when compared to health education, was not associated with any changes in leptin, or any of the other inflammatory biomarkers assessed [71]. These findings are not surprising given that leptin is produced by adipocytes adipose cells and is correlated with body fat and as meditation practices are unlikely to result in losses in body fat [69].

\section{Concluding Remarks and Future Perspectives}

Most studies to date have explored the effect of meditation practice on the HPA axis and much more research is needed to examine other aspects of the endocrine system. Whilst it is intriguing that various meditation practices appear to induce changes in endocrine function and consequently be associated with improvements in mental health, the underlying associations and mechanisms that might operate are unclear, though likely involve psychological, physiological and neurological processes. This is hampered by a lack of definition of what meditation is and the wide variety of forms of meditation practised. Better descriptions of actual practices and robust studies of their effect are needed. Many of the studies reported have small sample sizes, appear to have insufficient statistical power to demonstrate clear effects, and fail to include an active control group [9]. Thus, prime consideration should be given to the design, conduct and reporting of rigorously controlled trials to test the effectiveness of particular types of meditation practices in delineated populations, using well described interventions and comparison groups, and appropriate measures of outcome and experience. For example, studies should make explicit characteristics such as the precise nature of the meditation practice used (e.g. type, dose, duration, content), the person practising it (e.g. 
occupation, training, qualifications, experience), how and when it is practised (e.g. individual/group, audio, time) and where it is practised (e.g. home, gym, community/health centre). For example, did the study: offer individuals a choice of or preference for a particular form of meditation; measure the level of practice (e.g. some people may have completed the course but did not practice at home); or control for other factors like diet and exercise? Was the intervention too brief or dose too small to be able to produce a meaningful therapeutic effect on, for instance, endocrine function or mental health outcomes? To illustrate, for example, the effect of duration of meditation practice on biological measures, a recent study of a single 10-minute audio-guided mindfulness meditation suggested that such practice can promote effective heart rate regulation, and thereby effective recovery, after a stressful event for individuals with tension and migraine headaches [72]. In contrast, an earlier study of group-delivered transcendental meditation for 90 minutes a day for six months, compared to health education, found no statistically significant differences in brain natriuretic peptide and cortisol levels among individuals with heart failure [73]. In addition to trials, qualitative studies are also needed to explore how meditation practices might work, taking into account contextual factors such as culture, religion and beliefs, and help untangle this complex field and explain possible mechanisms. Overall, whilst this paper suggests there is a connection between meditation, the endocrine system and health and wellbeing, the area remains underexplored and awaits better understanding.

\section{Author Contributions}

M.C.P. conceptualized the outline. M.C.P., D.R.T., and C.S.F. discussed the content, drafted the manuscript, and approved the final version.

\section{Disclaimer Statement}

The authors have no conflicts of interest to disclose. 


\section{References}

1. Williams, J.M.G. and Kabat-Zinn, J. (2011) Mindfulness: diverse perspectives on its meaning, origins, and multiple applications at the intersection of science and dharma. Contemp. Buddhism 12, 1-18

2. Jevning, R., et al. (1992) The physiology of meditation: a review. A wakeful hypometabolic integrated response. Neurosci. Biobehav. Rev. 16, 415-424

3. Walsh, R. and Shapiro, S.L. (2006) The meeting of meditative disciplines and Western psychology: a mutually enriching dialogue. Am. Psychol. 61, 227-239

4. Lating, G.E.J. (2002) Meditation. In: A Clinical Guide to the Treatment of the Human Stress Response (Meichenbaum, D., ed), pp. 199-214, Springer; New York

5. Cahn, B.R. and Polich, J. (2006) Meditation states and traits: EEG, ERP, and neuroimaging studies. Psychol Bull. 132, 180-211

6. Fox, K.C., et al. (2014) Is meditation associated with altered brain structure? A systematic review and meta-analysis of morphometric neuroimaging in meditation practitioners. Neurosci. Biobehav. Rev. 43, 48-73

7. Fox, K.C., et al. (2016) Functional neuroanatomy of meditation: a review and meta-analysis of 78 functional neuroimaging investigations. Neurosci. Biobehav. Rev. 65, 208-228

8. Pascoe, M.C., et al. (2017) Yoga, mindfulness-based stress reduction and stress-related physiological measures: a meta-analysis. Psychoneuroendocrinoogy 86, 152-168

9. Pascoe, M.C., et al. (2017) Mindfulness mediates the physiological markers of stress: systematic review and meta-analysis. J. Psychiatr. Res. 95, 156-178

10. Levine, G.N., et al. (2017) Meditation and cardiovascular risk reduction: a scientific statement from the American Heart Association. J. Am. Heart Assoc. 6, e002218

11. Glaser, R. and Kiecolt-Glaser, J.K. (2005) Stress-induced immune dysfunction: implications for health. Nature Rev. Immunol. 5, 243-251

12. Lazarus, R. S. and Folkman, S. (1984) Stress, Appraisal, and Coping. Springer; New York

13. Chrousos, G.P. (2009) Stress and disorders of the stress system. Nat. Rev. Endocrinol. 5, 374381

14. Oken, B.S., et al. (2015) A systems approach to stress, stressors and resilience in humans. Behav. Brain Res. 282, 144-154

15. Greicius, M.D., et al. (2009) Resting-state functional connectivity reflects structural connectivity in the default mode network. Cereb. Cortex 19, 72-78

16. Broyd, S.J., et al. (2009) Default-mode brain dysfunction in mental disorders: a systematic review. Neurosci. Biobehav. Rev. 33, 279-296

17. Zhou, Y., et al. (2010) Increased neural resources recruitment in the intrinsic organization in major depression. J. Affect. Disord. 121, 220-230

18. Hamilton, J.P., et al. (2011) Default-mode and task-positive network activity in major depressive disorder: implications for adaptive and maladaptive rumination. Biol. Psychiatry 70, 327-333

19. Marchand, W.R. (2014) Neural mechanisms of mindfulness and meditation: evidence from neuroimaging studies. World J. Radiol. 6, 471-47

20. Garrison, K.A., et al. (2015) Meditation leads to reduced default mode network activity beyond an active task. Cogn. Affect. Behav. Neurosci. 15, 712-720

21. Ventriglio, A., et al. (2015) Early-life stress and psychiatric disorders: epidemiology, neurobiology and innovative pharmacological targets. Curr. Pharm. Des. 21, 1379-1387

22. Iwata, M., et al. (2013) The inflammasome: pathways linking psychological stress, depression, and systemic illnesses. Brain Behav. Immunity 31, 105-114

23. Salim, S., et al. (2012) Inflammation in anxiety. Adv. Protein Chem. Struct. Biol. 88, 1-25 
24. Silverman, M.N. and Sternberg, E.M. (2012) Glucocorticoid regulation of inflammation and its functional correlates: from HPA axis to glucocorticoid receptor dysfunction. Ann. N. Y. Acad. Sci. 1261, 55-63

25. Miller, D.B. and O'Callaghan, J.P. (2002) Neuroendocrine aspects of the response to stress. Metabolism 51, 5-10

26. Pascoe, M.C., et al. (2011) Inflammation and depression: why poststroke depression may be the norm and not the exception. Int. J. Stroke 6, 128-135

27. Ranabir, S. and Reetu, K. (2011) Stress and hormones. Indian J. Endocrinol. Metab. 15, 18-22

28. Sapolsky, R.M., et al. (2000) How do glucocorticoids influence stress responses? Integrating permissive, suppressive, stimulatory, and preparative actions. Endocrine Rev. 21, 55-89

29. Kinlein, S.A., et al. (2015) Dysregulated hyothalamic-pituitary-adrenal axis function contributes to altered endocrine and neurobehavioral responses to acute stress. Front. Psychiatry 6, 31.

30. Walton, K.G. and Levitsky, D.K. (2003) Effects of the transcendental meditation program on neuroendocrine abnormalities associated with aggression and crime. J. Offend. Rehabil. 36, 67-87

31. Manzaneque, J.M., et al. (2011) Psychobiological modulation in anxious and depressed patients after a mindfulness meditation programme: a pilot study. Stress Health 27, 216-222

32. Tang, Y.Y., et al. (2015) The neuroscience of mindfulness meditation. Nature Rev. Neurosci. $16,213-225$

33. Brand, S., et al. (2012) Influence of mindfulness practice on cortisol and sleep in long-term and short-term meditators. Neuropsychobiology 65, 109-118

34. Branstrom, R., et al. (2013) Effects of mindfulness training on levels of cortisol in cancer patients. Psychosomatics 54, 158-164

35. Hoge, E.A., et al. (2018) The effect of mindfulness meditation training on biological acute stress responses in generalized anxiety disorder. Psychiatry Res. 262, 328-332

36. Vandana, B., et al. (2011) Impact of integrated Amrita meditation technique on adrenaline and cortisol levels in healthy volunteers. Evid. Based Complement. Alternat. Med. 2011, 379645.

37. Jevning, R., et al. (1978) Adrenocortical activity during meditation. Horm. Behav. 10, 54-60

38. Walton, K.G., et al. (1995) Stress reduction and preventing hypertension: preliminary support for a psychoneuroendocrine mechanism. J. Alternat. Complement. Med. 1, 263-283

39. Fan, Y., et al. (2014) Cortisol level modulated by integrative meditation in a dose-dependent fashion. Stress Health 30, 65-70

40. Carlson, L.E., et al. (2007) One year pre-post intervention follow-up of psychological, immune, endocrine and blood pressure outcomes of mindfulness-based stress reduction (MBSR) in breast and prostate cancer outpatients. Brain Behav. Immunity 21, 1038-1049

41. Walton, K.G., et al. (2004) Lowering cortisol and CVD risk in postmenopausal women: a pilot study using the Transcendental Meditation program. Ann. N. Y. Acad. Sci. 1032, 211-215

42. Black, D.S., et al. (2017) Mindfulness practice reduces cortisol blunting during chemotherapy: A randomized controlled study of colorectal cancer patients. Cancer 123, 3088-3096

43. Raffone, A. and Srinivasan, N. (2009) An adaptive workspace hypothesis about the neural correlates of consciousness: insights from neuroscience and meditation studies. Prog. Brain Res. 176, 161-180

44. Raffone, A. and Srinivasan, N. (2010) The exploration of meditation in the neuroscience of attention and consciousness. Cogn. Process 11, 1-7

45. Cahn, B.R. and Polich, J. (2006) Meditation states and traits: EEG, ERP, and neuroimaging studies. Psychol. Bull. 132, 180-211 
46. Travis, F. and Shear, J. (2010) Focused attention, open monitoring and automatic selftranscending: categories to organize meditations from Vedic, Buddhist and Chinese traditions. Conscious. Cogn. 19, 1110-1118

47. Ortiga-Carvalho, T.M., et al. (2016) Hypothalamus-Pituitary-Thyroid Axis. Compr. Physiol. 6, 1387-1428

48. Kikuchi, M., et al. (2005) Relationship between anxiety and thyroid function in patients with panic disorder. Prog. Neuropsychopharmacol. Biol. Psychiatry 29, 77-81

49. Stipcevic, T., et al. (2008) Thyroid activity in patients with major depression. Coll. Antropol. 32, 973-976

50. Werner, O.R., et al. (1986) Long-term endocrinologic changes in subjects practicing the Transcendental Meditation and TM-Sidhi program. Psychosom. Med. 48, 59-66

51. MacLean, C.R.K., et al. (1997) Effects of the transcendental meditation program on adaptive mechanisms: changes in hormone levels and responses to stress after 4 months of practice. Psychoneuroendocrinology 22, 277-295

52. Chatterjee, S., Mondal S. (2017) Effect of combined yoga programme on blood levels of thyroid hormones: a quasi-experimental study. Indian J Tradition. Knowl. 16, 9-16

53. Chatterjee, S. and Mondal, S. (2014) Effect of regular yogic training on growth hormone and dehydroepiandrosterone sulfate as an endocrine marker of aging. Evid. Based Complement. Alternat. Med. 2014, 240581

54. Harinath, K., et al. (2004) Effects of Hatha yoga and Omkar meditation on cardiorespiratory performance, psychologic profile, and melatonin secretion. J. Alternat. Complement. Med. $10,261-268$

55. Simsch, C., et al. (2002) Training intensity influences leptin and thyroid hormones in highly trained rowers. Int. J. Sports Med. 23, 422-427

56. Krotkiewski, M., et al. (1984) The effect of acute and chronic exercise on thyroid hormones in obesity. Acta Med. Scand. 216, 269-275

57. Salehi, A. (2019) The effect of eight weeks yoga program on the thyroid function in middleaged women. J. Physical Activity Hormones 2, 63-74

58. Nilakanthan, S., et al. (2016) Effect of 6 months intense Yoga practice on lipid profile, thyroxine medication and serum TSH level in women suffering from hypothyroidism: a pilot study. J. Complement. Integr. Med. 13, 189-193

59. Atlas, S.A. (2007) The renin-angiotensin aldosterone system: pathophysiological role and pharmacologic inhibition. J. Manag. Care Pharm. 13, 9-20

60. Yang, G., et al. (1996) Angiotensin II - an important stress hormone. Biol. Signals 5, 1-8

61. Hafner, S., et al. (2012) To live alone and to be depressed, an alarming combination for the renin-angiotensin-aldosterone-system (RAAS). Psychoneuroendocrinology 37, 230-237

62. Michaels, R.R., et al. (1979) Renin, cortisol, and aldosterone during transcendental meditation. Psychosom. Med. 41, 50-54

63. Rosen, E.D. and Spiegelman, B.M. (2006) Adipocytes as regulators of energy balance and glucose homeostasis. Nature 444, 847-853

64. Schwartz, M.W., et al. (2000) Central nervous system control of food intake. Nature 404, 661-671

65. Woods, S.C., et al. (1985) Insulin: its relationship to the central nervous system and to the control of food intake and body weight. Am. J. Clin. Nutr. 42, 1063-1071

66. Chaiopanont, S. (2008) Hypoglycemic effect of sitting breathing meditation exercise on type 2 diabetes at Wat Khae Nok Primary Health Center in Nonthaburi province. J. Med. Assoc. Thai. 91, 93-98

67. Paul-Labrador, M., et al. (2006) Effects of a randomized controlled trial of transcendental meditation on components of the metabolic syndrome in subjects with coronary heart disease. Arch. Intern. Med. 166, 1218-1224 
68. Sivasankaran, S., et al. (2006) The effect of a six-week program of yoga and meditation on brachial artery reactivity: do psychosocial interventions affect vascular tone? Clin. Cardiol. 29, 393-398

69. Bernotiene, E., et al. (2006) The role of leptin in innate and adaptive immune responses. Arthritis Res. Ther. 8, 217

70. Malarkey, W.B., et al. (2013) Workplace based mindfulness practice and inflammation: a randomized trial. Brain Behav. Immunity 27, 145-154

71. Vaccarino, V., et al. (2013) Effect of meditation on endothelial function in Black Americans with metabolic syndrome: a randomized trial. Psychosom. Med. 75, 591-599

72. Azam, M.A., et al. (2016) Individuals with tension and migraine headaches exhibit increased heart rate variability during post-stress minfulness meditation practice but a decrease during a post-stress control condition - a randomized, controlled experiment. Int. J. Psychophysiol. $110,66-74$

73. Jayadevappa, R., et al. (2007). Effectiveness of transcendental meditation on functional capacity and quality of life of African Americans with congestive heart failure: a randomized control study. Ethn. Dis. 17, 72-77 


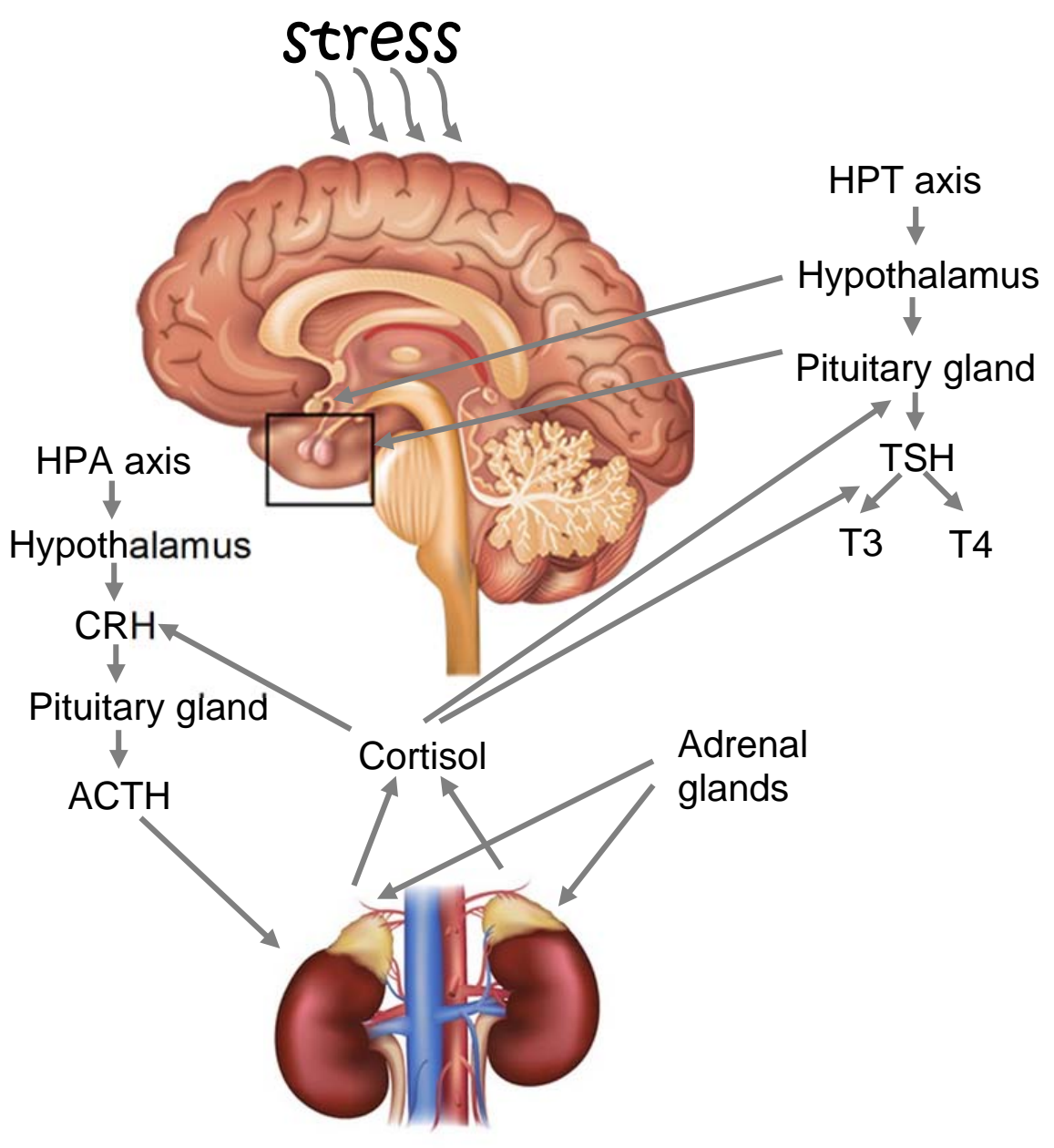

Figure 1. The role of the endocrine system in mediating stress 